\title{
Nondestructive inspection of aerospace composites by a fiber-coupled laser ultrasonics system
}

\author{
J.-F. Vandenrijt* , F. Languy, C. Thizy, M.P. Georges \\ Centre Spatial de Liège, Université de Liège, Liege Science Park, B-4031 Angleur, Belgium
}

\begin{abstract}
Laser ultrasonics is a technique currently studied for nondestructive inspection of aerospace composite structures based on carbon fibers. It combines a pulsed laser impacting the surface generates an ultrasound inside the material, through the nondestructive thermoelastic effect. Second a detection interferometer probes the impacted point in order to measure the displacement of the surface resulting from the emitted ultrasound wave and the echo coming back from the different interfaces of the structure. Laser ultrasonics is of interest for inspecting complex shaped composites. We have studied the possibility of using frequency doubled YAG laser for the generation and which is fiber-coupled, together with a fibercoupled interferometric probe using a YAG laser in the NIR. Our final system is a lightweight probe attached to a robot arm and which is able to scan complex shapes. The performances of the system are compared for different wavelengths of generations. Also we have studied some experimental parameters of interest such as tolerance to angle and focus distance, and different geometries of generation beams. We show some examples of inspection of reference parts with known defects. In particular C-scans of curved composites structures are presented.
\end{abstract}

Keywords: Laser-based Ultrasounds, Composite materials, Nondestructive inspection

\section{INTRODUCTION}

Laser ultrasonics ${ }^{[1][2]}$ is an interesting technique for the inspection of composite structures with complex shapes. The wider use of composite materials in the aerospace industries induces intensive research on adapted nondestructive inspection techniques. Ultrasonics is widely used in industry but requires either direct contact with the part inspected or to work at distance in water tank. For these reasons, optical contactless techniques are intensively studied, like active thermography ${ }^{[3][4]}$, shearography ${ }^{[5]}$ or combination of both ${ }^{[6][7]}$, which are imaging methods working directly on a large number of points. Nevertheless, some industrial domains are highly conservative, making these emergent techniques difficult to replace UT. Therefore there are important efforts to improve ultrasound testing (UT). Compared to classical UT, Laser UT (LUT) offers the advantage that no contact or couplant is needed and difficult to access points can be accessed by laser beams at distance. LUT usually combines two principles: the first one is the generation of an ultrasound wave at distance by a laser ${ }^{[2]}$, the second is the detection of ultrasound at distance by an interferometric system. For the generation, a pulsed laser beam insulates briefly the material on its surface which generates the ultrasound through thermoelastic nondestructive effect or through the destructive ablative effect. For industrial application on composites, the second one has to be avoided and the laser and its parameters must be correctly chosen to work in thermoelastic regime. The second principle is the detection of ultrasound at distance by an interferometric system with a large optical etendue allowing working with potentially scattering surfaces ${ }^{[2]}$. Various principles have been presented in literature. In any cases, a laser beam is sent on the surface to be probed and the ultrasound wave travelling to the surface will perturb the phase of the laser probe beam. These variations can be measured through a passive interferometer, like the confocal Fabry Perot (FP) ${ }^{[2]}$, or through adaptive interferometers based on the two-wave mixing (TWM) in photorefractive crystals ${ }^{[8]}$.

Our works aim at developing a robotized system addressing medium size composite parts provided by aerospace industries. These parts have generally a square meter size and complex shapes ${ }^{[9]}$. Industrial companies which are part of this project produce curved or assembled parts fully made of carbon fiber reinforced polymers (CFRP). Economic interest of LUT has been demonstrated for high end military aircraft composite parts and the question obviously arises in the civilian industries. Application cases have to be distinguished into medium or large parts, respectively smaller or larger than the square meter ${ }^{[10]}$. Large parts could be scanned at high speed with high repetition rate laser (a few $\mathrm{kHz}$ ), but this requires huge efforts in laser developments for both the generation and detection systems.

*jfvandenrijt@ulg.ac.be; phone +32-(0)4-3824661; www.csl.ulg.ac.be 
Our aim is to develop a medium cost industrial LUT system which can address medium size complex shaped CFRP parts. The idea is to have a flexible lightweight optical head which couples both the generation and detection laser beams. This optical head is interfaced on the flange of a 6-axis robot system that can be programmed to follow paths on complex structures. An obvious way to have flexibility is to work with laser beams transported by optical fibers, with all laser and detection equipment located at a remote location, a few meters away from the inspection working area. While a detection system fully fiber-coupled already exist, and is considered in our system, we show the use of a fiber-coupled solution for the generation, through a pulsed YAG Q-switch laser at $532 \mathrm{~nm}$. This solution is for the first time to our knowledge studied and compared to more classical approaches with other generation lasers.

In this paper, we will describe the development of the fiber-coupled LUT system. Also we will review the study of parameters of interest governing the performance of the technique. In particular we compare our generation wavelength with the more classical solution using a TEA $\mathrm{CO}_{2}$ laser. Also we briefly study the effect of geometry of beams and angle of incidence. At last, we will provide results of inspection of a curved shaped CFRP structure.

\section{DEVELOPMENT OF FIBER-COUPLED LASER ULTRASOUND SYSTEM}

Since we want to combine the generation and detection laser beams, a common way is to use two separate wavelengths and to incorporate a dichroic beamsplitter which transmits one wavelength and reflects the other one. The detection systems available on the market work either in the near infrared $(1.06 \mu \mathrm{m}$ or $1.55 \mu \mathrm{m})$ or in the green $(532 \mathrm{~nm})$. We have chosen the PDL laser proposed by Tecnar Company with the TWM detection probe (based on GaAs photorefractive crystal). This laser works at $1.06 \mu \mathrm{m}$ and is based on an MOPA configuration. This system is already full fiber-coupled and is delivered with a 10 meter robust flexible conduit which guides the different beams from remote sources and detectors on one side to a small lightweight optical probe on the other side. This system provides a high peak power of $500 \mathrm{~W}$ over pulse duration of $85 \mu \mathrm{s}$.

Concerning the generation, we needed to choose a cost-effective laser that is fiber-coupled and has a different wavelength than the detection. Literature shows that the efficiency of generating longitudinal waves in graphite/epoxy depends on the optical penetration depth, itself function of the wavelength ${ }^{11]}$. Furthermore, short pulses (in the nanosecond or tens of nanosecond range) are required. TEA $\mathrm{CO}_{2}$ lasers $(10.6 \mu \mathrm{m})$ are generally used in most LUT commercial systems because they industrialized and reliable ${ }^{[10][12]}$. However there is no optical fiber technology for such pulsed $\mathrm{CO}_{2}$ lasers and light is brought to the inspected part via mirrors in an articulated arm, which does not offer the flexibility of optical fibers. The best wavelengths for graphite/epoxy are small bands between 3.3 and $4 \mu \mathrm{m}$, which can be found in OPO lasers ${ }^{[13]}$. In principle they can be coupled to fibers but not commercial and cost-effective solution is offered at the moment and must constitute a development in itself. Therefore despite the great hopes of this solution, we discarded it. The other remaining wavelengths are those provided by YAG Q-switched lasers, either at $1.06 \mu \mathrm{m}$ or $532 \mathrm{~nm}$. We discarded $1.06 \mu \mathrm{m}$ because it corresponds to the detection wavelength. So we could not easily separate laser beams from detection and generation segments easily through a dichroic element. Consequently we have decided to work with generation at $532 \mathrm{~nm}$. The advantage of this wavelength is that some companies offer fiber-coupled YAG Qswitched laser with sufficient energy to be used for generation (a few tens of milliJoules). The laser we considered is the Ultra 50 from Quantel which is initially coupled to a 3 meter optical fiber interfaced through an SMA connector to the laser head. The laser works at $30 \mathrm{~Hz}$ repetition and allows $30 \mathrm{~mJ}$ at the output of the fiber. Because of this repetition rate, the repetition rate of the detection system was also set to $30 \mathrm{~Hz}$.

Figure 1 shows an overview of the whole setup. The pulsed detection laser (PDL) unit incorporates the long pulse laser which is split in two beams by a beamsplitter (BS). One beam is sent into the pump beam fiber (PBF) and constitutes the pump beam for the photorefractive crystal (PRC) of the TWM detection unit. The second beam is directly sent to the optical head. Figure 2 gives a detail sketch of the optical head which incorporates the detection optical probe provided by Tecnar. The latter contains the detection laser fiber (DLF) and the output beam is further collimated and passes through a frontal lens (FL) which focuses the illumination beam at a distance of $20 \mathrm{~cm}$. The light reflected by the object enters the optical probe through FL and is injected into the signal beam fiber (SBF) which transports the signal beam directly to the TWM detection unit (figure 1). In the TWM unit, the signal beam is mixed in the PRC with the pump beam. A differential photodetector (PD) module is placed in the signal beam after the PRC. The PDL laser allows long pulses of $80 \mu$ s duration with a repetition rate of $30 \mathrm{~Hz}$ and peak power of $300 \mathrm{~W}$. 
The generation laser is an Ultra 50 from Quantel which delivers $30 \mathrm{~mJ}$ at the output of a $3 \mathrm{~m}$ fiber specially developed by Quantel. We used this generation laser fiber (GLF) to develop the optical head which is shown in Figure 2 and which combines the detection and generation beams. Later the $3 \mathrm{~m}$ fiber has been replaced by a new $10 \mathrm{~m}$ long fiber no longer developed by Quantel but by Ceramoptec, with the same numerical aperture and core diameter as the former. This allows having the same length than the Tecnar flexible conduit and to attach the new GLF fiber to it and place the generation laser unit at the same remote location than the PDL unit. A computer controls the different units of the setup. The analog differential signal of the TWM unit is digitized by a high speed digitizer onboard a computer.

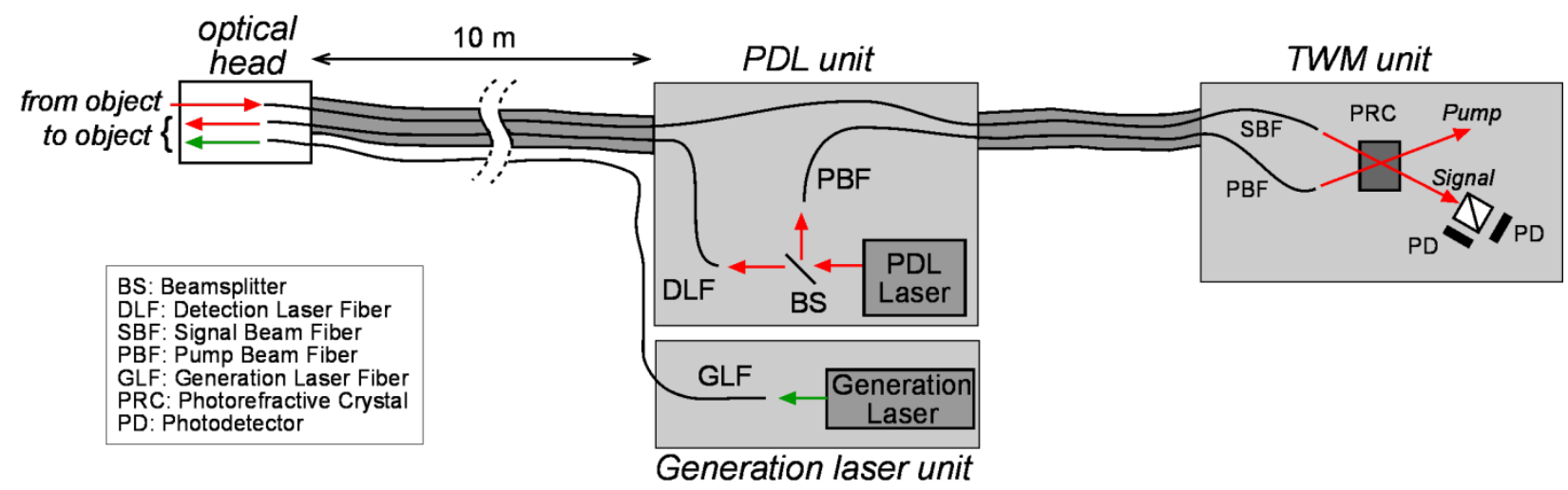

Figure 1. Overall scheme of the system.

Figure 2 shows a detail of the optical head which combines the green generation laser beam (GLB) and the detection probe. A dichroic beamsplitter (DBS) transmits the illumination and signal beam (SB) to and from the probe to the object. The GLB is made incident to the same object point via a folding mirror M and the DBS which reflects the $532 \mathrm{~nm}$ wavelengths. The lens $L$ position allows selecting the generation beam diameter. Although it is shown focused on the object, the GLB can be collimated or have different shapes. In the next section, we will show the study of generation beam shapes, among other parameters of interest.

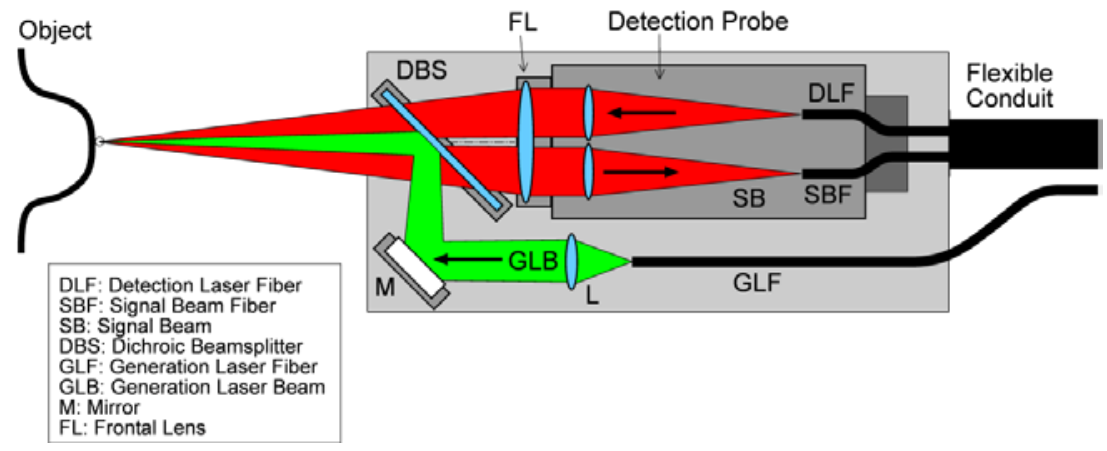

Figure 2. Sketch of the laser ultrasound optical head

\section{STUDY OF PARAMETERS OF INTEREST}

\subsection{Laser wavelength}

It is well known that the wavelength of the generation laser is of primary importance for the efficiency of the generation process in graphite/epoxy laminates (CFRP). The latter is due to thermoelastic effect and its efficiency is driven by the optical absorption. Some wavelengths are weakly absorbed by the resin and totally absorbed by the carbon fibers. Conversely some other wavelengths are well absorbed by resin which acts as a bulk source. Therefore the internal geometry of composite structures is of high importance for selecting the generation wavelength. Numerous studies have been performed in the past, comparing different available laser sources ${ }^{[12][14][15]}$. Mainly the $\mathrm{CO}_{2}$ laser line at $10.6 \mu \mathrm{m}$ and fundamental YAG line at $1.06 \mu \mathrm{m}$ were compared. It was found that the YAG line was more efficient for the 
generation, while the damage threshold for CFRP is smaller with YAG ${ }^{[12]}$. As already explained in the previous section, we have considered working at $532 \mathrm{~nm}$ for the generation for an easier separation with the detection beams through the optical head. From literature it is known that $532 \mathrm{~nm}$ has similar performances than $1.06 \mu \mathrm{m}^{[15]}$. However, compared to previous literature, there was no comparative study on true CFRP samples of interest.

Compared inspections on a CFRP sample has been performed with our system and another one which uses the same detector (Tecnar TWM) but a TEA $\mathrm{CO}_{2}$ laser from Light Machinery for the generation. The sample is shown in Fig3 (a), attached to a 6 -axis robot arm for the scanning. The sample has dimensions of $185 \times 145 \times 4.4 \mathrm{~mm}^{3}$, with 6 flat bottom holes: 3 of them have $12 \mathrm{~mm}$ diameter and 1, 2 and $3 \mathrm{~mm}$ depths, the 3 others have $6 \mathrm{~mm}$ diameter and 1,2 and $3 \mathrm{~mm}$ depths. These holes are arranged as shown in Fig3 (b). Fig3 (c) show the temporal signals (A-scans) obtained on the sample with the two systems. In both cases, the first pulse is seen on the left. It arises from the displacement of the surface measured by the TWM detection after the generation laser pulse has reached the surface. Then echoes are observed and due to the ultrasound longitudinal wave travelling from the surface to the back wall of the plate and returning to the surface. It can be seen that, in the case of the YAG $532 \mathrm{~nm}$ generation, multiple echoes are observed while with the $\mathrm{CO}_{2}$ laser, only the first echo is visible. The multiple echoes with YAG come from the different travels of the same ultrasound longitudinal wave between the front surface and the back one. Observing multiple echoes is an advantage if one wishes to better analyze the attenuation of ultrasound signal for detecting tiny variations, e.g. related to porosity.

(a)

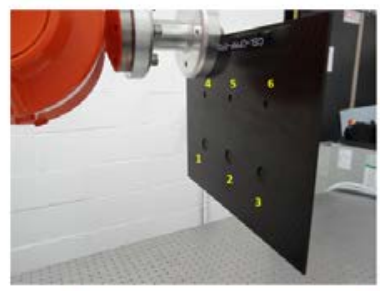

(b)
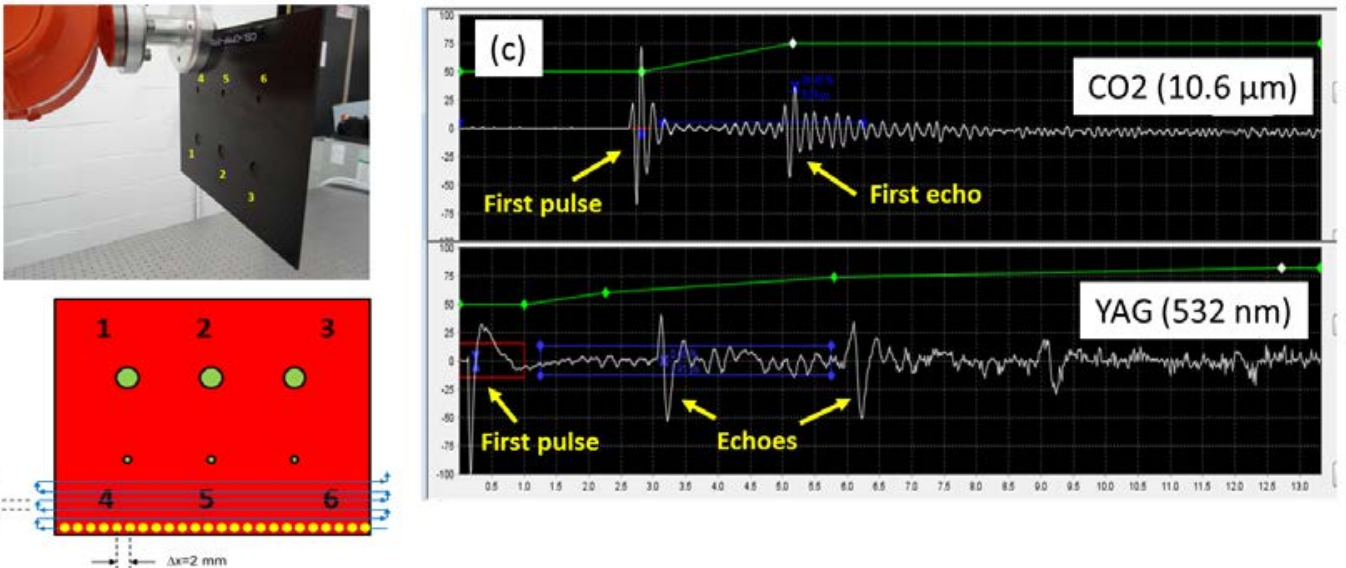

Figure 3. (a) CFRP sample with 6 flat bottom holes, (b), sketch of the sample with scanning strategy, (c) A-scans obtained with both wavelengths.

The A-scans of Fig3 (c) are accumulated during scanning of the CFRP sample and provide the so-called C-scans after two distinct post-processing. The first one consists in measuring the change of signal amplitude between the first pulse and the first echo. The second one consists in measuring the time interval between them (Time-of-Flight, TOF). The first one allows detecting density variations, change of materials, porosity, whereas the second one allows measuring variations of defect depths. They are both of interest in nondestructive inspection. Fig4 shows the amplitude and TOF Cscans obtained on the sample with both generation laser. In the TOF, a given color is associated with the time interval between the first pulse and the echo. The yellow color corresponds to the back wall echo. The scanning step is $1 \mathrm{~mm}$. It can be seen that contrast in attenuation is better with YAG, while they are roughly equivalent with TOF.

It can be seen on these results that the amplitude variations are better contrasted in the case with the YAG laser generation than with the $\mathrm{CO}_{2}$. Concerning the TOF, contrast is equivalent between both systems for the deeper defects (in the middle and on the right). Shallow defects (1 mm depth) are barely visible in both cases but at such depths, it is usually difficult to distinguish between both first pulse and first echo, which is a specificity of pulse-echo methods ("dead zone"). 


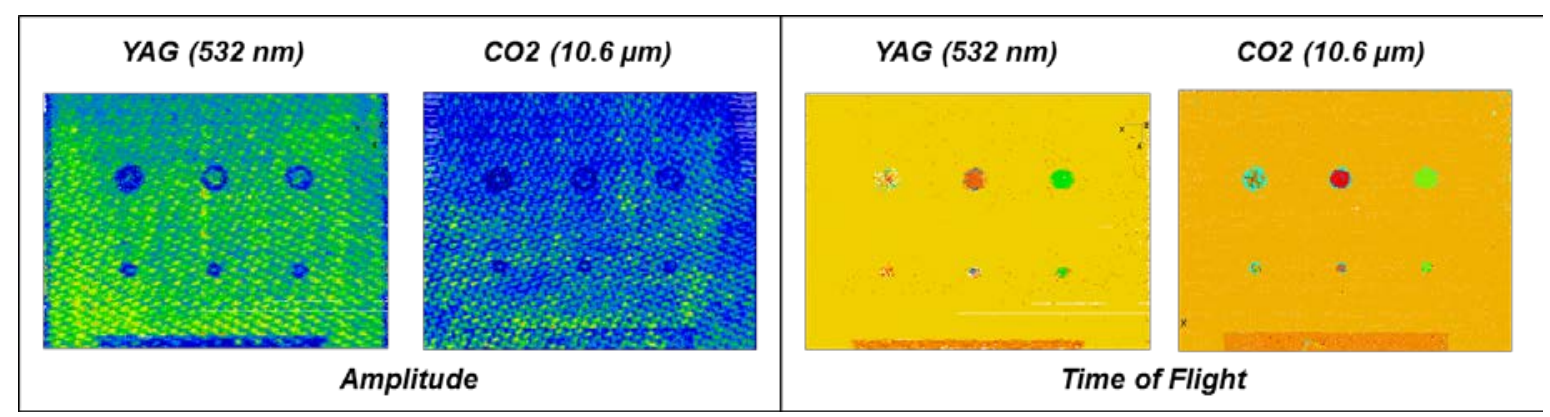

Figure 4. C-scans obtained in Amplitude and Time-of-Flight with two different generation wavelengths.

\subsection{Geometry of generation beam}

The propagation of ultrasound in materials appears in various directions from the surface to the rest of the structure. Mainly we are interested in longitudinal waves travelling perpendicularly to the surface. Another propagation mode is the bulk is the shear waves departing transversally from the longitudinal ones. Therefore if the generation area is slightly misaligned (on purpose or accidentally) from the inspection area, one could have variations in the signal. Therefore it could be of interest to see if the geometry of the generation beam has an impact on the resulting signal. We have considered different configurations, which are shown in Fig 5. The converging configuration (Fig 5(a)) is used most of time. It requires that the generation focusing point is located at the same place that the focusing point of the detection beam, as is shown in Fig 2. The second is collimated (Fig5 (b)) with a typical $10 \mathrm{~mm}$ diameter. It has the advantage over the first configuration of a larger tolerance to the distance, contrarily to the converging one which must be well aligned. Also when the generation is converging, it can lead to higher energy densities which can damage the sample surface. The third configuration is a ring (Fig5 (c)) which is formed by the combination of an axicon followed by a lens. The input beam of this assembly is supposed to be collimated after the generation fiber by a first lens L (see Fig2). The inner and outer diameters of the generation beam can be changed in function of lenses used in the combination.

(a)

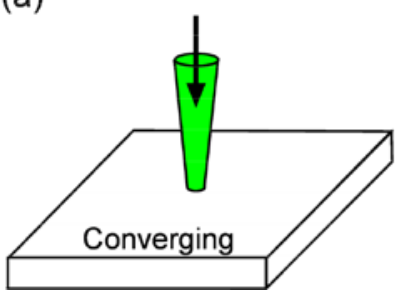

(b)

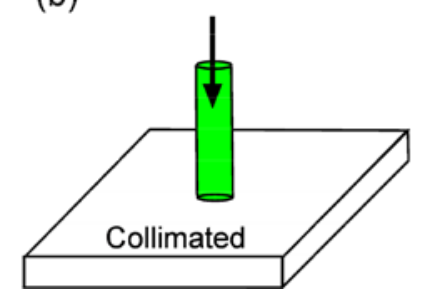

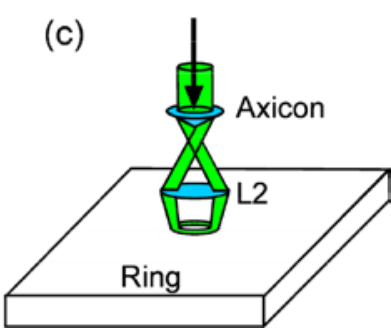

Figure 5. Different geometrical configurations for the generation laser beam

An optical bench has been used for studying the different configurations of generation beam, together with the detection (Fig6 (a)). The beams were also characterized with beam profiler. In particular, Fig6 (b) shows the beam profiles for the ring configuration.

(a)

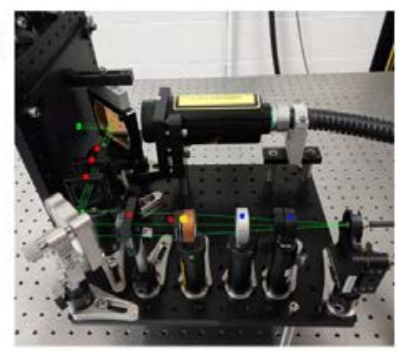

(b)

b) Beams characterization

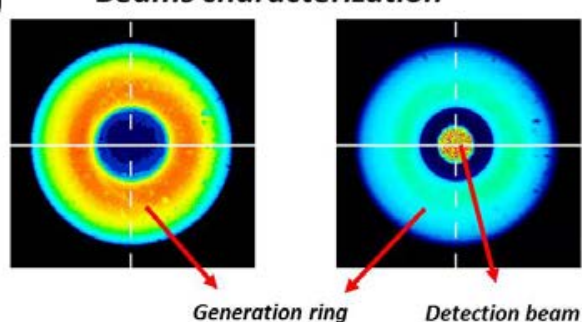

Figure 6. (a) Reconfigurable optical bench for studying the geometry of generation beam, (b) Typical beam profile of the ring configuration, without (left) and with (right) the detection in the center of the ring. 
Figure 7 shows typical A-scans obtained with the different configurations, for different parameters of lenses. From all these experiments, it was found that, globally, the collimated configuration is the best choice. First of all, it is the easiest to build and align. The collimated configuration also allowed observing the multiple echoes (what is interesting for integrating amplitude changes for measuring weak density variations in case of porosity), whereas this was not found for other cases. Also the non-collimated cases will suffer from any changes in the distance to the sample during the scanning process, while this won't be the case for the collimated one. Therefore, even if the non-collimated configurations have sometimes provided a better visibility of the first pulse and the first echo, this behavior can rapidly change if other geometric parameters change, such as the distance or the angle to the sample. Consequently we will consider the collimated configuration in all the experiments and inspections that will be presented later.
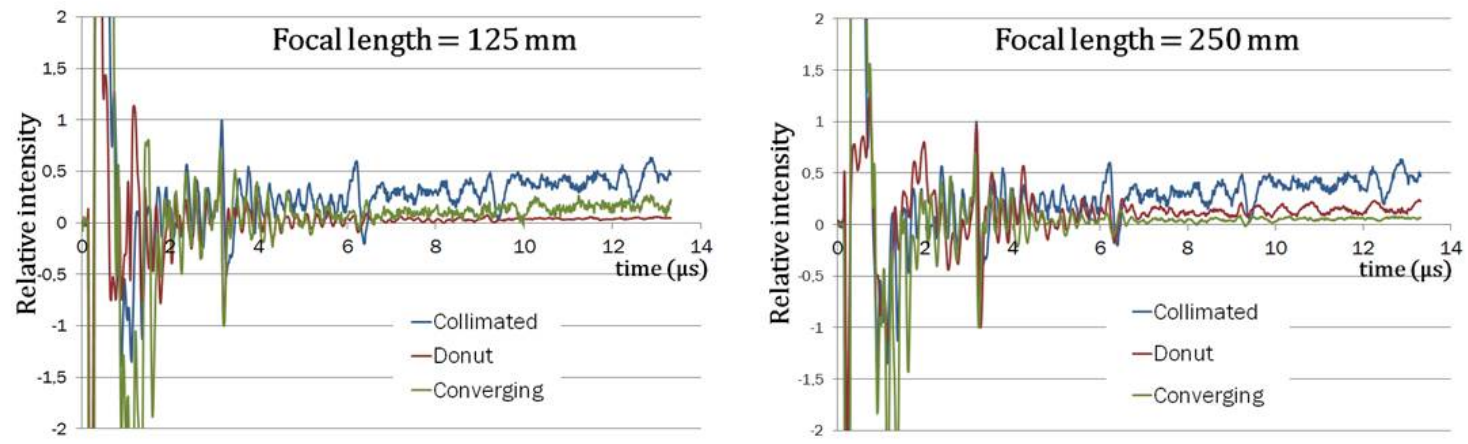

Figure 7. Typical A-scans obtained for different configuration and parameters of lenses used.

\subsection{Angle of incidence and distance to the sample}

The angle of incidence used for the inspection is of primary importance for practical implementation of LUT on realistic specimens. The reflectivity properties of the sample have a direct effect on the angle to be used for the inspection. As can be seen in Fig2, the beam of the detection system which is used to probe the surface (coming from DLF) is reflected back to the detection system through the combination of lenses and the SBF. Only a portion of the beam reflected by the surface forms the useful signal beam (SB) that is further used for the detection. If the object is mostly scattering, the SB will be weak but the tolerance on the incidence angle is expected large. On the contrary, if the object is mostly specular, the SB will be more intense, with a small tolerance on the incidence angle. The distance to the sample is also expected to induce effect on the reflected signal. Indeed, in the purely specular case, working at a distance equal to the focal distance of the frontal lens FL of the detection probe (see Fig2) will allow the reflected beam (SB) to enter the reception fiber. In the purely scattering case, one expects a higher tolerance to distance. However in such defocused situation less light is expected to enter the fiber.

We have performed an experiment based on the collimated generation beam and the converging detection system. Different samples have been considered, representative of what can be found in composite industries. The surface properties of these samples are clearly different from one another and result from the large variety of processes that are used to build these composite structures. Although we have not measured the roughness of these samples, they were sufficiently and clearly different visually from one another with respect to their reflectivity properties.

Fig 8(a) shows a CFRP sample, similar to the one used in section 3.1 (Fig 3(a)), except with a single flat bottom hole. This sample has a medium roughness. Fig 8(b) has a high roughness, with small scale bumps covering the surface. It represents what is usually called the "vacuum bag side" of a composite part, due to the manufacturing process. Fig 8(c) shows the other part of the sample, but with a high specular reflectivity. It is represents what is usually called the "tool side" because this part of the sample was in contact to a flat metallic surface during the manufacturing process. All these three different surfaces of interest are surrounded in different colors.

In order to assess the effect of the incidence angle, we used a goniometric arm, with its center of rotation placed on one point of the surface, at the best focal distance to the optical detection probe. Then the angle was varied by steps of 0.5 deg and the intensity of the first pulse is measured. The fit of the results for the 3 types of surfaces is shown in Fig 8(d). It can be shown that the most specular sample (blue curve) is the less tolerant to the angle, while the roughest one (red curve) has the largest tolerance, with the intermediate case with medium tolerance. Also we can have similar behavior in 
the case of the distance to the sample, as is shown in Fig 8(e), where the distance with respect to the best focus of the detection system has been varied, at normal incidence.
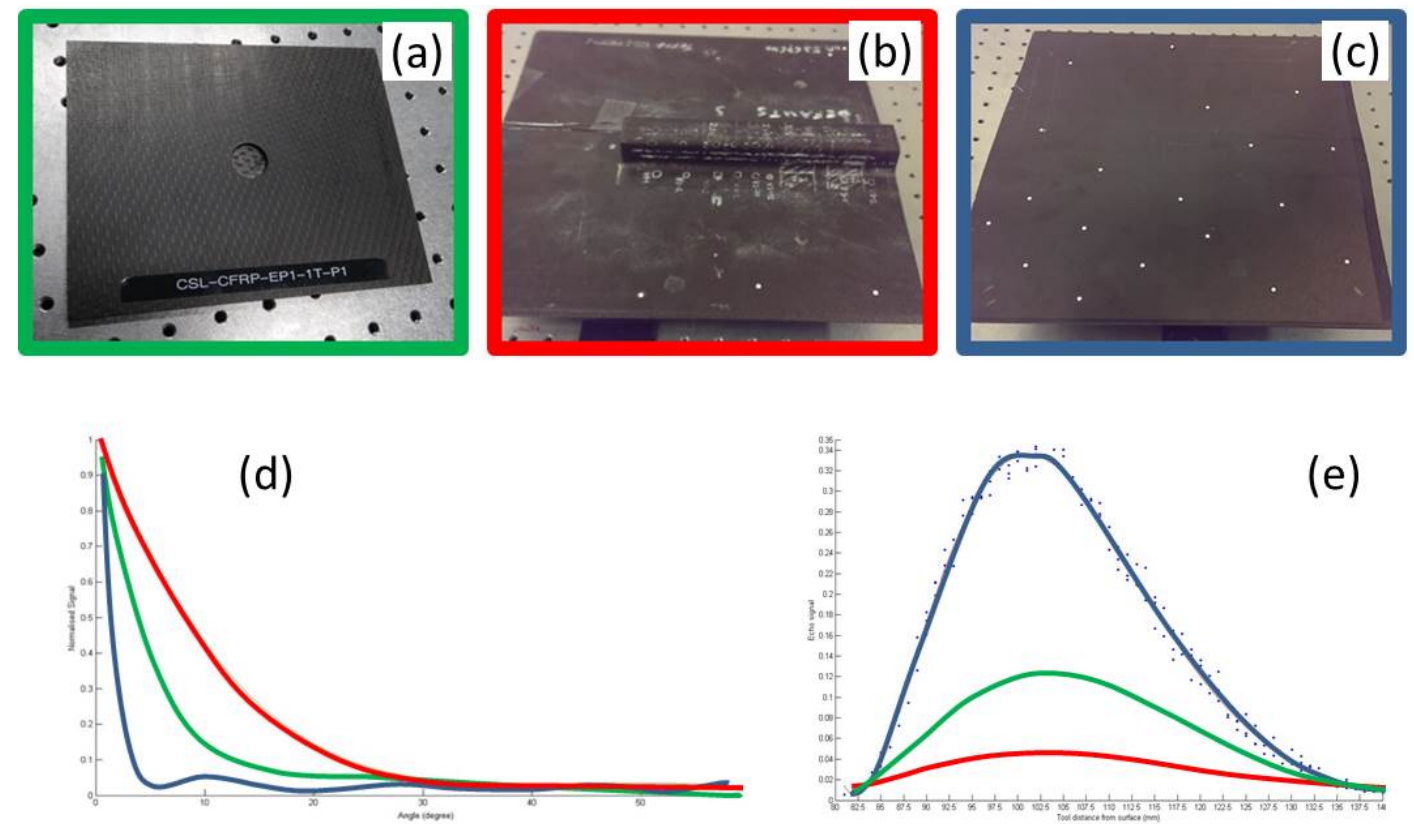

Figure 8. (a) CFRP sample with medium roughness, (b) with high roughness, (c) with low roughness, (d) effect of incidence angle for the 3 cases, (e) effect of the distance.

The conclusion of this study is that, if one wishes to obtain the best intensity of the signal beam, we need to take care of the reflectivity properties of the samples and adapt the scanning system. In our case, the optical head is attached to a 6axis robot arm. If we now the geometry of the structure to scan, we can define paths for the scan in a way that the axis of the optical head is perpendicular to the surface and the optical head kept at a fixed distance from the surface.

\section{INSPECTION OF COMPLEX SHAPED CFRP STRUCTURES}

The fully fiber-coupled LUT system developed with a collimated $532 \mathrm{~nm}$ pulsed laser for generation and TWM detection at $1064 \mathrm{~nm}$ has been used in various inspections. A good example is shown in Fig 9. A portion of slat is shown at Fig 9(a). It is a made with monolithic CFRP skins, arranged in a curved shape, with a stiffener with a $\mathrm{T}$ profile in the inner part of the structure. Artificial defect zones have been created between the skin and the stiffener. They are marked on the surface by white lines. Fig 9(b) shows the LUT system in position in front of the sample. The scan has been performed with a sampling distance of $2 \mathrm{~mm}$ in every direction, keeping the angle normal to the surface and the distance fixed from the surface (a few $\mathrm{cm}$ ).

After compilation of A-scans in all points, a post-processing allows forming C-scans, both in amplitude (Fig 9(c)) and time-of-flight (Fig 9(d)). The TOF displays in false color the time interval between the first pulse and the first echo, coming back from the back wall of the skins (dark blue), the back wall of the upper part of the T profile of the stiffener (medium green). One can see a change of color in the skin signal, turning from dark blue to yellow. This is due to the higher thickness in the most curved part of the sample. Also the signal from the interface with the $\mathrm{T}$ profile turns from green to red. The different circular defects (debonding between the skin and the stiffener) are easily seen as different color patches in the stiffener signal. Rotation of the 3D C-scans allows best viewing the different defects (Figs 9 (e-f)). 

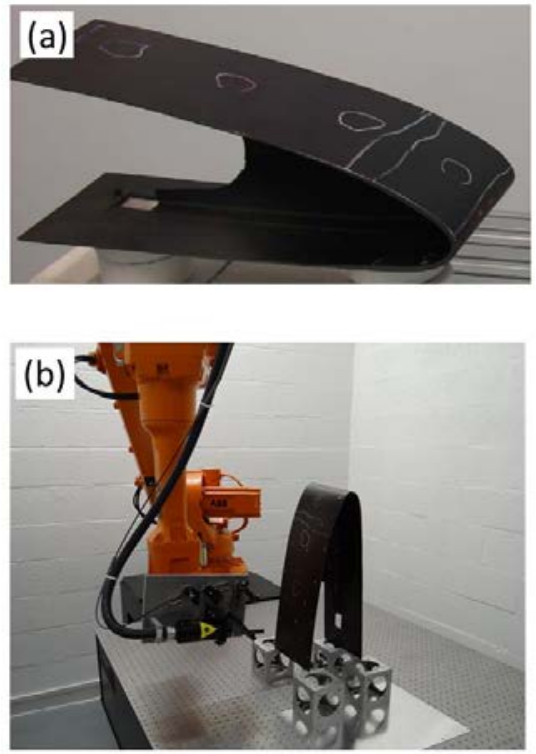

(c) C-Scan Amplitude

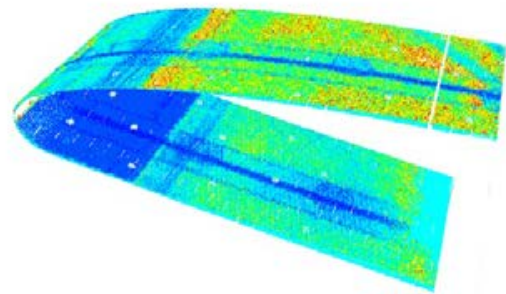

(d) C-Scan Time of Flight

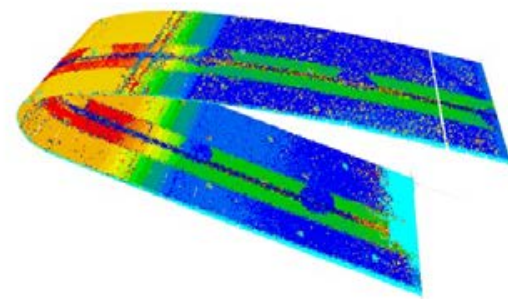

(e)

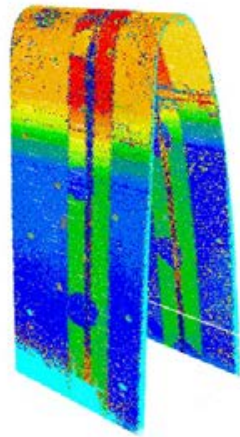

(f)

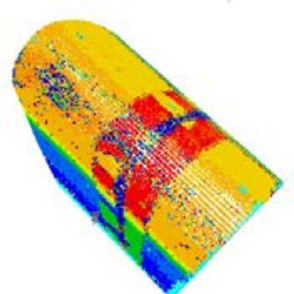

Figure 9. Inspection of complex shaped composite structure. (a) Specimen, (b) During the scan, (c)-(d) C-scan, resp. in amplitude and time-of-flight, (e)-(f) different perspective angles of the time-of-flight C-Scan.

\section{CONCLUSION}

We have shown the development of a laser ultrasonic inspection system for complex shaped CFRP structures. The system combines two segments. The first one is an interferometer for the detection of ultrasound at distance with a fibercoupled detection system based on the two-wave mixing with photorefractive crystals at $1064 \mathrm{~nm}$, with a YAG laser with long pulses. The second one is a YAG Q-switch nanosecond pulsed laser working in the green and which has been coupled in an optical fiber. We have developed an optical head which allows coupling both beams. This system is attached to a robot arm which allows scanning of curved shaped structures. It is the first time to our knowledge that combination of green generation and infrared detection is reported. We have studied some parameters of interest on the basis of true industrial composite samples. First we have compared the green laser generation with the more usual one at $10.6 \mu \mathrm{m}$ (TEA $\mathrm{CO}_{2}$ laser). We have seen that we obtained excellent results with our green laser, although at much smaller cost and higher flexibility, compared to $\mathrm{CO}_{2}$ generation. Indeed no fiber coupling solutions exist for the latter. Afterwards we have shown the effect of geometry of generation beam by comparing converging, collimated and ring configurations. We concluded that the collimated one is the easiest to deal with. Also the effect of incidence angle and distance of the optical head to the surface has been studied. It is shown that tolerance of the performances to these parameters strongly depends on the surface reflectivity type (between purely specular to purely scattering). At last we have shown an example of LUT C-scans, both in amplitude and time-of-flight, for the inspection of a complex CFRP structure with artificial defects.

\section{ACKNOWLEDGMENTS}

These results have been obtained with financial support of Wallonia under the project TECCOMA (contract $\mathrm{N}^{\circ}$ 7281) in the frame of SKYWIN competitiveness pole. We would like to thank Mr Julien Walter and Tommy Brouillette from the Centre Technologique en Aérospatiale (St-Hubert, QC) for access to their LUT system which helped us to compare our system and theirs working with a CO2 laser for the generation. Authors also acknowledge the financial support of bilateral cooperation project from Wallonie-Bruxelles International and Quebec Ministery of International Relations and Francophony. 


\section{REFERENCES}

[1] Scruby, C.B. and Drain, L.E., [Laser Ultrasonics. Techniques and Applications], Adam Hilger, Bristol, New York, Philadelphia, (1990)

[2] Monchalin, J-P., "Laser-ultrasonics: from the laboratory to industry," AIP Conference Proceedings 700, 3-31 (2004)

[3] Maldague, X., [Theory and Practice of Infrared Technology for Nondestructive Testing], Wiley-Interscience (2001).

[4] Jorge, I., Venegas, P., Vega, L., Lopez, I., Vollheim, B., Krausz, L., \& Georges, M., "Review of thermal imaging systems in composite defect detection, " Infrared Physics \& Technology 61, 167-175 (2013)

[5] Kalms, M., and Osten, W., "Mobile shearography system for the inspection of aircraft and automotive components," Opt. Eng. 42 (5), 1188- 1196 (2003)

[6] Georges, M.P., "Comparison between thermographic and holographic techniques for nondestructive testing of composites: similarities, differences and potential cross-fertilization," Proc. SPIE 9660, 966002 (2015)

[7] Georges, M.P., "Speckle interferometry in the long-wave infrared for combining holography and thermography in a single sensor. Applications to nondestructive testing: The FANTOM Project," Proc. SPIE 9525, 952557 (2015)

[8] Blouin, A., and Monchalin, J-P., "Detection of ultrasonic motion of a scattering surface by two-wave mixing in a photorefractive GaAs crystal," Appl. Phys. Letters 65, 932-934 (1994)

[9] Kalms, M., Focke O. and von Kopylow, C., "Applications of Laser Ultrasound NDT method on Composite structures in Aerospace Industry," Proc. SPIE 7155, 71880E (2008)

[10] Voillaume, H., Simonet, D., Brousset, C., Barbeau, P., Arnaud, J.-L., Dubois, M., Drake, T., and Osterkamp, M., "Analysis of commercial aeronautics applications of laser ultrasonics for composite manufacturing," Proc. $9^{\text {th }}$ Eur. Conf. on NDT (ECNDT2006), We.1.1.1 (2006), http://www.ndt.net/article/ecndt2006/doc/We.1.1.1.pdf

[11] McKie, A., and Addison, R., "Practical considerations for the rapid inspection of composite materials using laser-based ultrasound, " Ultrasonics 32, 333-345 (1994)

[12] Stratoudaki, T., Edwards, C., Dixon, S., and Palmer, S., "The role of epoxy resin in the mechanism of laser generated ultrasound in carbon fiber reinforced composites, " Proc. SPIE 5046, 89-98 (2003)

[13] Lorraine, P., Dubois, M., Bauco, A.S., and Filkins, R., "A new laser source for ultrasound generation in composites," AIP Conference Proceedings, 295-301 (2000)

[14] Dubois, M., Lorraine, P., Venchiarutti, B., Bauco, A.S., Filkins, R., Drake, T., and Yawn, K., "Optimization of temporal profile and optical penetration depth for laser-generation of ultrasound in polymer-matrix composites," AIP Conference Proceedings 509, 287-294 (2000)

[15] Dubois, M., Chuang, S.-Y., Lorraine, P., Drake, T., Yawn, K. and Filkins, R., "Progress on the development of an advanced laser ultrasound generation source for inspection polymer-matrix composites," AIP Conference Proceedings 615, 300-307 (2002) 